Brief Report

\title{
The role of every-day executive function in social impairment and adaptive skills in Autism Spectrum Disorder with intellectual disability
}

\author{
Stella Tsermentseli*, Jeannette Farago Tabares, Evangelia Chrysanthi Kouklari \\ Department of Psychology, Social Work \& Counselling, University of Greenwich, London, UK
}

\section{A R T I C L E I N F O}

Number of reviews done is 2

Keywords:

ASD

Intellectual disability

Executive function

Adaptive functioning

\begin{abstract}
A B S T R A C T
Background: Although executive function (EF) deficits are a recognised component of the cognitive phenotype of Autism Spectrum Disorder (ASD), particularly in children without general intellectual delay, little is known about ecological measures of EF and their outcome correlates among individuals with ASD and co-occurring intellectual disability. This exploratory study examined every-day EF in the classroom among children and adolescents diagnosed with both ASD and intellectual disability (ASD-ID) and their correlations with social impairment and adaptive functioning.

Method: Teachers of 40 children and adolescents diagnosed with ASD-ID completed the Behavior Rating Inventory of Executive Function, the Vineland Adaptive Behavior Scales, and the Social Responsiveness Scale.

Results: A global executive dysfunction profile was found in ASD-ID, with most prominent deficits occurring in shifting. Results also showed that metacognitive executive processes predicted adaptive communication skills above and beyond IQ and social impairment in ASD-ID.

Conclusions: Our findings corroborate a specific metacognitive executive function-adaptive communication association in ASD. EF interventions might be important treatment targets for improving functioning, especially in the communicative domain, in ASD-ID.
\end{abstract}

\section{Introduction}

In order to organise one's self and effectively respond to the environment, executive function (EF) is necessary. EF refers to a set of higher-order cognitive control processes, such as inhibition, shifting, organisation, planning, self-monitoring, and working memory (Anderson, 1998; Gioia, Isquith, \& Guy, 2001). Deficits in EF are common and have been conceptualised as an associated cognitive feature in several Autism Spectrum Disorder (ASD) samples (Demetriou et al., 2018; Hill, 2004). While not a core symptom of ASD, EF has been shown to be a predictor of key social and behavioural outcomes (Kanne et al., 2011; Pellicano, 2010). Despite research having addressed the EF impairments of individuals with ASD, questions remain.

First, the literature on EF in ASD is dominated by utilisation of performance-based EF tasks. Cross-sectional studies on children and adolescents with ASD, using performance-based EF measures, have revealed significant deficits in shifting/flexibility, planning, working memory and inhibition aspects (for a review see Kenworthy, Yerys, Anthony, \& Wallace, 2008). These studies though have only focused on ASD individuals with normal intellectual ability (IQ > 70). Intellectual Disability (ID) has been found in an estimated 31\% of children with ASD in a recent report (Developmental Disabilities Monitoring Network Surveillance Year 2010 Principal

\footnotetext{
* Corresponding author.

E-mail address: s.tsermentseli@gre.ac.uk (S. Tsermentseli).
} 
Investigators; Centers for Disease Control and Prevention (CDC), 2014). This means that there is currently a lack of knowledge on how EF is reflected in the ASD population with ID (ASD-ID).

In addition, a recent meta-analysis (Demetriou et al., 2018) showed that performance-based EF measures did not achieve clinical utility in differentiating between ASD and typical controls, while informant-based ecological measures such as the Behavior Rating Inventory of Executive Function (BRIEF) (Gioia, Isquith, Guy, \& Kenworthy, 2000) achieved clinical marker criteria. This lends further support to the proposition that measures with ecological validity, commonly known as "every-day" EF rating scales, may be more appropriate in clinical practice. A possible advantage of ecologically valid EF assessments is their potential utility for individuals with co-occurring ASD and ID who may find it difficult to complete performance-based EF tests. Most importantly performance-based EF tasks include additional language and cognitive demands that might exacerbate EF deficits in those individuals.

Based on ratings from the BRIEF across development, it has been found that every-day EF deficits in ASD without ID were prominent across all subdomains, with a peak difficulty noted in shifting (Granader et al., 2014; Wallace et al., 2016). Nevertheless, very little work to date has examined the profiles of every-day EF among individuals with ASD-ID. One exception is the work of Panerai, Tasca, Ferri, Genitori D'Arrigo, \& Elia (2014) who found that individuals with ASD-ID showed shared EF deficits to ASD without ID. Panerai et al. (2014) however only recruited 8 individuals with ASD-ID, and therefore utilisation of the BRIEF on a larger sample is needed to establish an executive dysfunction profile of ASD-ID.

Another open field of investigation is the relation between every-day EF, social impairment and adaptive functioning. Thus far, there is contradicting evidence regarding whether every-day EF is associated with social impairment in ASD without ID. For example, Leung, Vogan, Powell, Anagnostou, and Taylor (2016) demonstrated a distinct metacognitive EF-social impairment link in ASD, whilst Kenworthy, Black, Harisson, Della Rossa, and Wallace (2009) found no link between BRIEF and social symptoms. No study to date has investigated such association in ASD-ID. With respect to adaptive behaviour, Gilotty, Kenworthy, Sirian, Black, and Wagner (2002) reported that EF deficits in metacognition domain were important predictors of maladaptive skills in ASD. The nature of the relationship was extended by Pugliese et al. (2015) reporting significant cross-sectional and longitudinal associations between BRIEF scores and adaptive functioning in children and adolescents with ASD. Wallace et al. (2016) also found that both behavioural regulation and metacognition were important predictors of adaptive functioning for adults with ASD. However, Panerai et al. (2014) in the only study to date to include ASD-ID in their sample $(\mathrm{N}=8)$ demonstrated contradictory findings as no correlations were found between BRIEF and adaptive skills within the ASD group in their study. Studies have not yet been published exploring these relationships in a pure ASD-ID sample; whether the same pattern of results would be found amongst individuals with ASD-ID remains to be explored.

The primary focus of this exploratory study is thus to fill in the theoretical breach that previous research has created, hence the focus on those individuals within ASD-ID. To the authors' best knowledge, the present study is the first to examine EF profiles employing ecological every-day EF measures among a sample of children and adolescents with ASD-ID. The second aim was to investigate the role of every-day EF on social impairment and adaptive skills within ASD-ID. It was predicted that ASD-ID children and adolescents would show deficits in EF domains relative to the population mean. It was also expected that shifting would be the greatest weakness within this group. Finally, we hypothesised that impaired EF aspects, especially in metacognition, would be associated to social impairment and maladaptive skills.

\section{Methods}

\subsection{Participants}

Participants were 40 children and adolescents, diagnosed with ASD and comorbid ID, aged between 6 and 16 years (12 females). All children possessed a formal diagnosis of intellectual disability and received a score of $<70$, as determined by the abbreviated version of the Wechsler Intelligence scales (two subtests: vocabulary and matrix reasoning; Wechsler, 1999) (see Table 1 for details). All participants also held an official ASD diagnosis by a qualified clinician using DSM-IV (Diagnostic and Statistical Manual of Mental Disorders, 4th Edition) or DSM-V (5th edition) criteria (American Psychiatric Association (APA), 1994, 2013), and qualified for a

Table 1

Participant characteristics $(\mathrm{N}=40)$ including mean age, IQ as well as executive function index scores, adaptive functioning and social impairment scores.

\begin{tabular}{llll}
\hline Measure & M & SD & Range (Minimum-Maximum) \\
\hline Age & 9.20 & 3.44 & $6-16$ \\
Full scale IQ & 52.80 & 7.10 & $46-68$ \\
SRS & 120.23 & 27.36 & $69-179$ \\
BRIEF BRI & 79.63 & 18.24 & $58-118$ \\
BRIEF MI & 77.23 & 11.82 & $60-111$ \\
VABS socialisation & 63.33 & 12.47 & $42-103$ \\
VABS communication & 57.77 & 13.11 & $31-86$ \\
VABS daily living & 54.63 & 12.33 & $23-78$ \\
\hline
\end{tabular}

Note. SRS = Social Responsiveness Scale; BRIEF = Behavior Rating Inventory of Executive Function; BRI = Behavioural Regulation Index; MI = Metacognition Index; VABS $=$ Vineland Adaptive Behavior Scales. 
"broad ASD" on the Autism Diagnostic Interview/Autism Diagnostic Interview-Revised (Le Couteur et al., 1989; Lord, Rutter, \& Le Couteur, 1994) and/or the Autism Diagnostic Observation Schedule (Lord, Rutter, DiLavore, \& Risi, 2000). Children were in receipt of an Education, Health and Care Plan (EHCP), formerly known as Statement of Special Educational Needs. Exclusion criteria included the presence of a diagnosed psychiatric illness or any known comorbid medical conditions, as these may cloud interpretations of what is driving observed difficulties.

\subsection{Measures}

\subsubsection{Executive function}

Teachers completed the Behavior Rating Inventory of Executive Function-Teacher Report (BRIEF-TR; Gioia et al., 2000) for each child in their class participating in the study. The BRIEF measures two broad areas/indices of EF: behavioural regulation (BRI), the ability to shift and modulate emotions and behaviour; and metacognition (MI), the ability to cognitively self-manage tasks and monitor performance. The BRI consists of three subscales (i.e., Inhibition, Shifting, and Emotional Control) and the MI consists of five subscales (i.e., Initiation, Working Memory, Planning/Organisation, Task Monitor, and Organisation of Materials). Raw scores are expressed as $T$ scores $(M=50 ; S D=10)$ derived from comparisons with normative age expectations. Higher scores are indicative of more EF difficulties and $T$ scores of 65 or higher are categorised as clinically significant.

\subsubsection{Social impairment}

Teachers also completed the Social Responsiveness Scale (SRS; Constantino \& Gruber, 2005), which is a 65-item rating scale. Responses to items are on a 4-point Likert scale with higher SRS total T scores indicative of greater social impairment. The SRS can be used as a screening tool (Constantino \& Gruber, 2005). All participants had a T score $>65$, corroborating the official ASD clinical diagnosis.

\subsubsection{Adaptive functioning}

Teachers also completed the Vineland Adaptive Behavior Scales (VABS-T; Sparrow, Cicchetti, \& Balla, 2005) for each child from their class who participated in the study. The VABS assess adaptive behavioural skills in socialisation, communication, and daily living of individuals. Standard scores were obtained for each domain. Higher scores suggest better adaptive skills.

\subsection{Procedure}

Participants were recruited from two schools specialised in ASD children with the highest level of autistic needs in North London, UK. The high SRS scores possibly represent a sample of children with severe level of social impairment. Two special education teachers filled the questionnaires for each child. Teachers were familiar with the children and had known them for at least one year. Ethical approval for the study was obtained, and all participants' parents/carers gave written informed consent (consistent with the Declaration of Helsinki) in compliance with the University Research Ethics Committee.

\subsection{Statistical analysis}

Analyses were conducted using IBM SPSS Statistics, version 24. Prior to conducting primary analyses, data were screened and no outliers were detected. Variables were also checked for normality and homogeneity assumptions of parametric tests. One-sample $t$ tests were run to examine the degree of the impairment on the BRIEF-TR scores of the ASD-ID group, relative to the population mean of 50. Furthermore, a repeated-measures ANOVA was run to examine the profile of EF scores within ASD-ID. Pearson's correlations were run to examine the association between EF indices and outcome variables (i.e. social impairment (SRS score) and adaptive behaviour (VABS communication, daily living and socialisation scores)). Based on results of correlations, factors were selected for inclusion in subsequent hierarchical multiple regression analyses.

\section{Results}

\subsection{Profile of executive function}

At the index score level, 93 and $90 \%$ of the sample exhibited a clinically significant impairment (i.e., a $T$ score $\geq 65$ ) on the BRIEF BRI and MCI, respectively. A series of one-sample $t$-tests first showed that children and adolescents with ASD-ID presented deficits on all domains of EF relative to the population mean of $50\left(p_{\mathrm{s}}<.05\right)$ and that the composite BRI index score $(M=79.63, S D=18.24)$ was not significantly higher compared to the composite $\mathrm{MI}$ index score $(M=77.23, S D=11.82, t(39)=1.045, p=.30)$. In order to further examine which EF domain presented the most prominent deficits within ASD-ID, a Repeated measures ANOVA including all 8 sub-scales was run, revealing a variable EF profile $(F(7,413)=1018.84, p<.001)$. Post-hoc pairwise comparisons, using Bonferroni correction to adjust for multiple comparisons from the BRI showed that the shifting score $(M=82.73, S D=19.91)$ was significantly higher than the score of inhibition $(M=72.33, S D=16.35, p<.001)$. Emotional control score $(M=77.37, S D=14.47)$ was also significantly higher than inhibition score $(M=72.33, S D=16.35, p<.05)$. Shifting score $(M=82.73, S D=19.91)$ was finally found also significantly higher compared to emotional control score $(M=77.37, S D=14.47, p<.05)$. These findings suggest that shifting impairment was the highest weakness within the BRI composite of BRIEF. On the other hand, the scores from the MI were 
Table 2

Correlation matrix between potential covariates, executive function, social impairment and adaptive skills scores for children and adolescents with ASD-ID.

\begin{tabular}{|c|c|c|c|c|c|c|c|}
\hline Measures & FSIQ & SRS & BRIEF MI & BRIEF BRI & VABS Socialisation & VABS Communication & VABS Daily Living \\
\hline Age & -.20 & .09 & $.58^{\text {sktk }}$ & $.68^{* * *}$ & -.14 & -.16 & -.14 \\
\hline FSIQ & - & .45 & -.31 & -.22 & .01 & $.46^{* * k}$ & $.46^{*}$ \\
\hline SRS & - & - & .14 & .12 & $-.70^{* *}$ & $-.58^{\text {st.ke }}$ & $-.40^{*}$ \\
\hline BRIEF MI & - & - & - & $.89^{* k *}$ & -.34 & $-.37^{*}$ & -.34 \\
\hline BRIEF BRI & - & - & - & - & .19 & -.20 & .00 \\
\hline VABS Socialisation & - & - & - & - & - & $.63^{* * x / 2}$ & $.52^{* k *}$ \\
\hline VABS Communication & - & - & - & - & - & - & $.73^{* *}$ \\
\hline
\end{tabular}

Note. FSIQ = Full scale IQ; SRS = Social Responsiveness Scale; BRIEF = Behavior Rating Inventory of Executive Function; BRI = Behavioural Regulation Index; MI = Metacognition Index; VABS = Vineland Adaptive Behavior Scales.

$$
* p<.01 \text {. }
$$

$* * p \leq .001$.

more evenly spread as the analysis showed non-significant differences between the 5 sub-domains when compared to each other within ASD-ID $(p>.05)$.

\subsection{Relations between EF, social impairment and adaptive skills}

Preliminary bivariate correlations (Table 2) indicated that age was not significantly correlated with outcome measures (SRS, VABS subdomain scores), whilst IQ was significantly correlated with adaptive communication and daily living. The BRIEF BRI and MI indices were not correlated with SRS scores, and therefore, further regression models predicting SRS scores were not conducted. Because SRS total score was correlated with adaptive skills, it was treated as a control variable in subsequent regressions. The BRIEF MI scores were significantly correlated with the VABS communication subdomain. BRI did not correlate with any outcome measures. Further inspection of individual BRIEF subscales showed that working memory $(r=.406, p=.01)$ as well as planning/organisation $(\mathrm{r}=.430, \mathrm{p}=.01)$ correlated with communication skills. No other significant correlations were found.

Hierarchical multiple regression analysis was conducted to identify the unique contribution of BRIEF MI to adaptive communication skills over and above social impairment as well as age and FSIQ. The results showed that the first block introducing age, FSIQ and SRS contributed significantly to the variance of the adaptive communication domain, $F(3,39)=8.39, p<.01$, and explained $47.8 \%$ of the variance. For BRIEF MI entered in Block 2, the total variance explained rose to $51.2 \%$, representing a significant increase of $3.4 \%, F(4,39)=6.35, p<.01$, of additional variance explained.

Since working memory and planning/organisation were correlated with adaptive communication skills, a second set of regressions was run to examine the contribution of these specific BRIEF subscales to adaptive communication skills after controlling for age, FSIQ and SRS. The results showed that the first block introducing age, FSIQ and SRS contributed significantly to the variance of the adaptive communication domain, $F(3,39)=8.39, p<.01$, and explained $47.8 \%$ of the variance. For BRIEF working memory and planning/organisation entered together in block 2 the total variance explained rose to $50.1 \%$, representing a significant increase of $2.3 \%(F(5,39)=5.07, p<.01)$ of additional variance explained. Our results suggest that fewer EF problems on the MI and particularly on the working memory subscale of the BRIEF were significant predictors of better adaptive communication skills. Full results of the hierarchical regression analyses are presented in Table 3.

\section{Discussion}

This study is presented as a first attempt to explore the profile of ecological measures of EF in people with ASD-ID. In agreement with previous studies documenting every-day EF difficulties in children and adolescents with ASD without ID, the present study confirms that based on teacher-report of EF in the classroom the peak weaknesses in ASD-ID is also in shifting. The present findings also found that only the metacognition (MI) index was predictive of adaptive communication skills above and beyond social impairment, age and FSIQ.

Based on teachers' reports of every-day EF functioning in the classroom, children and adolescents with ASD-ID exhibited global EF difficulties relative to the population mean. This finding is in line with previous research where BRIEF scores in children with ASD without ID were elevated across scales (Gioia, Isquith, Retzlaff, \& Espy, 2002; Granader et al., 2014). Closer examination of the profile of EF subdomains revealed particular difficulties in Shift subscale from the BRI. This pattern is also similar to that found amongst children and adolescents with ASD without ID (e.g., Granader et al., 2014). Overall, our results suggest that there is a similar profile of EF deficits in ASD-ID across the constructs examined, with the exception of shifting/cognitive flexibility.

Our findings demonstrate a relation between executive processes and adaptive functioning in children and adolescents with ASDID. In contrast, SRS scores were not associated with any EF skill. The latter is in agreement with Kenworthy et al. (2009), possibly indicating that poor performance on every-day EF tasks in ASD may reflect factors that are not directly related to social symptoms. Social impairment though correlates with adaptive functioning and might influence the EF-adaptive behaviour relationship, which has not been controlled for in previous studies. With regards to the link between EF and adaptive skills, our findings are largely 
Table 3

Adaptive communication skills scores regressed onto age, FSIQ, social impairment and executive function scores.

\begin{tabular}{|c|c|c|c|}
\hline Predictors & B & $\Delta \mathrm{R}^{2}$ & $t$ \\
\hline \multicolumn{4}{|l|}{ BRIEF index analyses } \\
\hline Step 1 & & $0.478^{* * *}$ & \\
\hline Age & -0.14 & & -0.43 \\
\hline FSIQ & 0.71 & & $2.61^{*}$ \\
\hline SRS & -0.25 & & -3.69 \\
\hline Step 2 & & $0.034^{\text {*t*k }}$ & \\
\hline BRIEF MI & -0.19 & & $-2.41^{*}$ \\
\hline \multicolumn{4}{|l|}{ BRIEF subscale analyses } \\
\hline Step 1 & & $0.478^{\text {*t*k }}$ & \\
\hline Age & -0.14 & & -0.43 \\
\hline FSIQ & 0.71 & & $2.61^{*}$ \\
\hline SRS & -0.25 & & $-3.69^{* * * *}$ \\
\hline Step 2 & & $0.023^{* * *}$ & \\
\hline BRIEF Working Memory & -0.47 & & $-2.07^{*}$ \\
\hline BRIEF Plan/Organise & -0.23 & & -0.99 \\
\hline
\end{tabular}

Note. FSIQ = Full scale IQ; SRS = Social Responsiveness Scale; BRIEF = Behavior Rating Inventory of Executive Function; MI = Metacognition Index.

$* p<.05$.

$* * p \leq .01$.

consistent with the extant literature. Indeed we found that the MI index of BRIEF only was related to communication skills above and beyond social symptoms and IQ. These results support previous findings where it was found that metacognition was significantly correlated with the communication subdomain of adaptive skills (Gilotty et al., 2002; Pugliese et al., 2015).

The present exploratory study presented some limitations. First a small convenience sample was used. Another limitation was the absence of a control group. Future studies that compare EF profiles in different ASD samples and typically developing children may build upon the findings of the current one. It would also be highly informative to compare individuals with ASD-ID to individuals with ID without ASD in order to better understand the nature of the relations between EF and adaptive functioning and symptoms. The evaluation of the EF profiles in ASD-ID using only teacher report EF ratings was another limitation of this study. Finally, the present research has focused on the effect of EF, IQ and ASD traits on adaptive skills, however the inclusion of different variables measuring co-morbid disorders such as ADHD, which is very common within the ASD population, could have revealed other possible predictors of maladaptive skills. Future studies should also investigate the role of language functioning (receptive and expressive) to the reported relation between EF and adaptive functioning in ASD-ID. Finally, since only metacognitive abilities were significant predictors of adaptive functioning, the present findings should be interpreted with caution as clear conclusions about the true association of social outcomes to the wider EF context in ASD-ID cannot be drawn.

Our findings of every-day EF problems and demonstration of associations between EF impairments and adaptive communication skills in ASD-ID suggest that EF is an important treatment target among children and adolescent with ASD-ID. Indeed, interventions that target metacognitive skills have been shown to improve communication abilities in children and adolescents with ASD (Kenworthy et al., 2014). Interestingly, Kenworthy et al. (2014) suggested that intelligence did not predict response to treatment, however as with most EF studies in ASD, only ASD without ID participants were included. Whether the same pattern of results is found in ASD-ID remains to be answered. If EF-based interventions also show to enhance treatment outcomes in ASD-ID, this would provide valuable clinical insights and also inform us about underlying mechanisms among these constructs.

\section{Conflict of interest}

All authors declare that they have no conflict of interests.

\section{Acknowledgements}

The authors are grateful for the Vice-Chancellor PhD Scholarship from the University of Greenwich. The authors would like to acknowledge their gratitude to the participating schools and children.

\section{References}

American Psychiatric Association (APA) (1994). Diagnostic and statistical manual of mental disorders (4th ed.). Washington, DC: Author.

American Psychiatric Association (APA) (2013). Diagnostic and statistical manual of mental disorders (5th ed.). Washington, DC: Author.

Anderson, V. (1998). Assessing executive functions in children: Biological, psychological, and developmental considerations. Neuropsychological Rehabilitation, 8, 319-349.

Constantino, J. N., \& Gruber, C. P. (2005). Social responsiveness scale (SRS). Los Angeles: Western Psychological Services.

Demetriou, E. A., Lampit, A., Quintana, D. S., Naismith, S. L., Song, Y. J. C., Pye, J. E., et al. (2018). Autism spectrum disorders: A meta-analysis of executive function. Molecular Psychiatry, 23(5), 1198-1204. http://dx.doi.org/10.1038/mp.2017.75. 
Developmental Disabilities Monitoring Network Surveillance Year 2010 Principal Investigators (2014). Prevalence of autism spectrum disorder among children aged 8 years-Autism and developmental disabilities monitoring network, 11 sites, United States, 2010. Morbidity and Mortality Weekly Report: Surveillance Summaries, $63(2), 1-21$.

Gilotty, L., Kenworthy, L., Sirian, L., Black, D. O., \& Wagner, A. E. (2002). Adaptive skills and executive function in autism spectrum disorders. Child Neuropsychology, 8(4), 241-248.

Gioia, G. A., Isquith, P. K., Guy, S. C., \& Kenworthy, L. (2000). Behavior rating inventory of executive function: BRIEF. Odessa, FL: Psychological Assessment Resources.

Gioia, G. A., Isquith, P. K., \& Guy, S. C. (2001). Assessment of executive function in children with neurological impairments. In R. Simeonsson, \& S. Rosenthal (Eds.). Psychological and developmental assessment (pp. 317-356). New York: The Guilford Press.

Gioia, G. A., Isquith, P. K., Retzlaff, P. D., \& Espy, K. (2002). Confirmatory factor analysis of the Behavior Rating Inventory of Executive Function (BRIEF) in a clinical sample. Child Neuropsychology, 8, 249-257. http://dx.doi.org/10.1076/chin.8.4.249.13513.

Granader, Y., Wallace, G. L., Hardy, K. K., Yerys, B. E., Lawson, R. A., Rosenthal, M., et al. (2014). Characterizing the factor structure of parent reported executive function in autism spectrum disorders: The role of cognitive inflexibility. Journal of Autism and Developmental Disorders, 44, 3056-3062.

Hill, E. L. (2004). Evaluating the theory of executive dysfunction of autism. Developmental Review, 24, $189-233$.

Kanne, S. M., Gerber, A. J., Quirmbach, L. M., Sparrow, S. S., Cicchetti, D. V., \& Saulnier, C. A. (2011). The role of adaptive behavior in autism spectrum disorders: Implications for functional outcome. Journal of Autism and Developmental Disorders, 41(8), 1007-1018.

Kenworthy, L., Yerys, B. E., Anthony, L. G., \& Wallace, G. L. (2008). Understanding executive control in autism spectrum disorders in the lab and in the real world. Neuropsychology Review, 18(4), 320-338.

Kenworthy, L., Black, D. O., Harrison, B., Della Rosa, A., \& Wallace, G. L. (2009). Are executive control functions related to autism symptoms in high-functioning children? Child Neuropsychology, 15(5), 425-440.

Kenworthy, L., Anthony, L. G., Naiman, D. Q., Cannon, L., Wills, M. C., Werner, M. A., et al. (2014). Randomized controlled effectiveness trial of executive function intervention for children on the autism spectrum. Journal of Child Psychology and Psychiatry, 55, 374-383.

Le Couteur, A., Rutter, M., Lord, C., Rios, P., Robertson, S., Holdgrafer, M., et al. (1989). Autism diagnostic interview: A standardized investigator-based instrument. Journal of Autism and Developmental Disorders, 19, 363-387.

Leung, R. C., Vogan, V. M., Powell, T. L., Anagnostou, E., \& Taylor, M. J. (2016). The role of executive functions in social impairment in autism spectrum disorder. Child Neuropsychology, 22(3), 336-344.

Lord, C., Rutter, M., \& Le Couteur, A. (1994). Autism diagnostic interview-revised: A revised version of a diagnostic interview for caregivers of individuals with possible pervasive developmental disorders. Journal of Autism and Developmental Disorders, 24, 659-685.

Lord, C., Rutter, M., DiLavore, P. C., \& Risi, S. (2000). Autism diagnostic observation schedule (ADOS). Los Angeles, California: Western Psychological Services.

Panerai, S., Tasca, D., Ferri, R., Genitori D'Arrigo, V., \& Elia, M. (2014). Executive functions and adaptive behaviour in autism spectrum disorders with and without intellectual disability. Psychiatry Journal, 1-11.

Pellicano, E. (2010). Individual differences in executive function and central coherence predict developmental changes in theory of mind in autism. Developmental Psychology, 46(2), 530-544.

Pugliese, C. E., Anthony, L., Strang, J. F., Dudley, K., Wallace, G. L., \& Kenworthy, L. (2015). Increasing adaptive behavior skill deficits from childhood to adolescence in autism spectrum disorder: Role of executive function. Journal of Autism and Developmental Disorders, 45(6), 1579-1587.

Sparrow, S. S., Cicchetti, V. D., \& Balla, A. D. (2005). Vineland adaptive behavior scales (2nd ed.). Circle Pines, MN: American Guidance Service.

Wallace, G. L., Kenworthy, L., Pugliese, C. E., Popal, H. S., White, E. I., Brodsky, E., et al. (2016). Real-world executive functions in adults with autism spectrum disorder: Profiles of impairment and associations with adaptive functioning and co-morbid anxiety and depression. Journal of Autism and Developmental Disorders, 46.

Wechsler, D. (1999). Wechsler abbreviated scale for intelligence. San Antonio, TX: The Psychological Corporation. 